\title{
Efficacy of neoadjuvant endocrine therapy compared with neoadjuvant chemotherapy in pre-menopausal patients with oestrogen receptor-positive and HER2-negative, lymph node-positive breast cancer
}

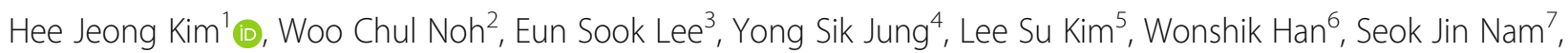
Gyung -Yub Gong ${ }^{8}$, Hwa Jung Kim ${ }^{9}$ and Sei Hyun Ahn ${ }^{1^{*}}$

\section{Abstract}

Introduction: Neoadjuvant endocrine therapy (NET) has demonstrated efficacy in post-menopausal patients with hormone-responsive breast cancer. This trial was designed to compare the efficacy of neoadjuvant chemotherapy (NCT) with NET in pre-menopausal breast cancer.

Patients and methods: In this prospective, randomised, phase III study, oestrogen receptor (ER)-positive, HER2negative, and lymph node-positive pre-menopausal breast cancer patients were recruited from 7 hospitals in South Korea. Enrolled patients were randomly assigned (1:1) to receive 24 weeks of either NCT or NET with goserelin and tamoxifen. The primary purpose was to evaluate the non-inferiority of NET compared to NCT using clinical response, assessed by MRI. Besides, pathological complete response rate ( $\mathrm{pCR})$, changes in Ki-67 expression, breast conservation surgery (BCS) rate, and quality of life were included as secondary endpoints.

Results: A total of 187 patients were assigned to receive NCT $(n=95)$ or NET $(n=92)$, and 87 patients in each group completed treatments. More NCT patients had complete response or partial response than NET patients using MRI (NCT $83.7 \%$ vs. NET 52.9\%, 95\% Cl 17.6-44.0, $p<0.001$ ) and callipers (NCT 83.9\% vs. NET 71.3\%, 95\% Cl 0.4-24.9, $p=$ 0.046). Three NCT patients (3.4\%) and one NET patient (1.2\%) showed PCR $(p<0.005)$. No difference existed in the conversion rate of BCS (13.8\% for NCT vs. $11.5 \%$ for NET, $p=0.531)$ and Ki-67 change $(p=0.114)$ between the two groups. Nineteen NCT patients had treatment-related grade 3 or worse events compared with none in the NET group. Conclusions: Better clinical responses were observed in pre-menopausal patients after 24 weeks of NCT compared to those observed after NET.

(Continued on next page)

\footnotetext{
*Correspondence: ahnsh@amc.seoul.kr

This study was presented as a poster discussion at the 2017 American Society of Clinical Oncology annual meeting.

1 Department of Surgery, University of Ulsan College of Medicine, Asan Medical Center, 88 Olympic ro 43 gil, song pa gu, Seoul 138-736, South Korea

Full list of author information is available at the end of the article
}

C C The Author(s). 2020 Open Access This article is licensed under a Creative Commons Attribution 4.0 International License, which permits use, sharing, adaptation, distribution and reproduction in any medium or format, as long as you give appropriate credit to the original author(s) and the source, provide a link to the Creative Commons licence, and indicate if changes were made. The images or other third party material in this article are included in the article's Creative Commons licence, unless indicated otherwise in a credit line to the material. If material is not included in the article's Creative Commons licence and your intended use is not permitted by statutory regulation or exceeds the permitted use, you will need to obtain permission directly from the copyright holder. To view a copy of this licence, visit http://creativecommons.org/licenses/by/4.0/ The Creative Commons Public Domain Dedication waiver (http://creativecommons.org/publicdomain/zero/1.0/) applies to the data made available in this article, unless otherwise stated in a credit line to the data. 
(Continued from previous page)

Trial registration: Clinicaltrials.gov, NCT01622361. Registration June 19, 2012.

Keywords: Neoadjuvant endocrine therapy, Neoadjuvant chemotherapy, Pre-menopausal women, Breast cancer, Clinical response

\section{Background}

For hormone-responsive breast cancer, the question of which patients can safely be spared adjuvant chemotherapy has been extensively studied. For cases involving hormone receptor-positive tumours, younger patients $(<$ 35 years old) show a poorer prognosis than older patients in terms of tamoxifen resistance [1]. However, this finding does not indicate that young patients should receive chemotherapy. The greater effects of chemotherapy on younger patients are likely to be partially explained by the endocrine effects of chemotherapy on ovarian function. Irrespective of the chemotherapy agent administered, iatrogenic amenorrhea is associated with an increased survival rate in oestrogen receptive (ER)-positive breast cancer [2, 3], and amenorrhea is a surrogate marker for effective treatment in hormone receptor-positive pre-menopausal breast cancer patients [4]. This finding suggests that irrespective of chemotherapy or endocrine therapy, amenorrhea is important for pre-menopausal patients who have an ER-positive, HER2negative tumour. The trial assigning individualized options for treatment (TAILORx) and the EORTC 10041/BIG 03-04 (MINDACT) studies can identify patients with a low-risk profile, thereby justifying the omission of chemotherapy based on its potentially low benefit [5-8].

Treatment decisions based on demographic characteristics and tumour burden have the potential to overtreat many individuals and undertreat others. The Oncotype DX assay has been studied for lymph node-positive breast cancer patients, and the SWOG 8818 study reported that the low-risk Oncotype DX group gained no additional benefits from chemotherapy [9]. SOFT and TEXT joint analysis showed excellent survival data that included lymph node-positive, ER-positive breast cancer patients who received endocrine therapy alone. These findings suggested that not all lymph node-positive, ER-positive, and HER2-negative tumours require chemotherapy. Apart from the tumour burden, identifying robust biological predictors of benefit is likely to be informative and useful. It has been shown that endocrine therapy, which can substitute for chemotherapy even though there is a high tumour burden in hormone-responsive breast cancer, is effective. The factors that determine chemotherapy sensitivity remain poorly understood and might not entirely overlap with negative predictors of endocrine sensitivity or prognostic determinants $[10,11]$.

Neoadjuvant treatment has benefits, which include reducing the tumour burden and evaluating the treatment response of the tumour. The response to neoadjuvant chemotherapy (NCT) is diverse and varies according to the intrinsic subtype. ER-positive, HER2-negative breast cancer shows a reduced pathologically complete response (pCR) rate, but better survival than other subtypes. Moreover, $\mathrm{pCR}$ is not related to survival in the luminal A subtype [12]. Therefore, the goal of neoadjuvant treatment for hormone-responsive tumours is to evaluate the treatment effects and reduce the tumour size prior to treatment, although it can rarely achieve pCR. Neoadjuvant endocrine therapy (NET) has been mostly studied for post-menopausal breast cancer patients in comparison with tamoxifen and aromatase inhibitors [13-15]. Semiglazov et al. compared NET and chemotherapy for post-menopausal breast cancer patients and found a similar clinical response between the two therapies [16]. The STAGE study was conducted for pre-menopausal breast cancer patients and compared gonadotropin-releasing hormone agonist (GnRHa) with tamoxifen and GnRHa with an aromatase inhibitor [17]. However, no clinical trial has yet compared NCT with NET for pre-menopausal patients.

Therefore, we hypothesised the non-inferior response of NET compared with NCT and reported a phase III clinical trial to compare the response between NCT and NET in pre-menopausal women with ER-positive, HER2-negative, and lymph node-positive breast cancer.

\section{Methods/design \\ Study design}

This is a phase III, open-label, prospective, randomised, multicentre, neoadjuvant study of chemotherapy versus endocrine therapy in pre-menopausal patient with hormone-responsive, HER2-negative, lymph nodepositive breast cancer [NEST]. Seven centres belonging to the Korean Breast Cancer Society Group (KBCSG012) participated in this study. The study protocol has been approved by the Korea Food and Drug Administration (KFDA), as well as the institutional review board of every trial centre, and was conducted in accordance with the Declaration of Helsinki, Good Clinical Practice, and the applicable local regulatory requirements on bioethics. For safety issue, the response rate of both groups was planned to be monitored. An independent data monitoring committee (IDMC) was established to monitor the study progress. The IDMC was responsible for deciding whether to continue the study when 66 patients in each treatment group completed their treatment 
regimens. This trial has been registered in ClinicalTrials. gov as number NCT01622361.

\section{Patients}

Eligible patients included pre-menopausal women with histologically confirmed ER-positive, HER2-negative, and lymph node-positive primary breast cancer. Lymph node positivity was required to be proven histologically prior to the start of treatment with core needle biopsy or fine needle aspiration. All patients were aged between 20 and 50 years. Pre-menopausal status was defined based on the following: (1) last menses within 6 months of randomisation and (2) for patients who have had hysterectomy, $\mathrm{E} 2 \geq 20 \mathrm{pg} / \mathrm{ml}$ and $\mathrm{FSH}<30 \mathrm{mIU} / \mathrm{ml}$ within 4 weeks of randomisation. ER positivity was defined as Allred score $\geq 3$ or modified Allred score $\geq 4$. Patients with inflammatory breast cancer, bilateral breast cancer, evidence of distant metastasis, or other malignancy were excluded. Written informed consent was obtained from all participants.

\section{Randomisation}

Patients were enrolled by the study investigators. Patients were randomly assigned (1:1) to receive either NCT or NET for 24 weeks prior to surgery. Block randomisation was performed using the e-CRF system, with patients stratified by the treating centre and clinical stage (stages II and III). Patients who signed an informed consent form entered screening and were assigned a unique patient screening number. Patients who completed the screening process and met all eligibility criteria were randomised through a central randomisation website. No patients were enrolled or began treatment prior to randomisation, and the assignment of a randomisation number was undertaken using the website. Adverse events were recorded at every patient visit and assessed according to the Common Terminology Criteria for Adverse Events Version 3.0.

\section{Procedures}

Patients were randomly assigned (1:1) to receive either adriamycin and cyclophosphamide $\left(60 \mathrm{mg} / \mathrm{m}^{2}\right.$ adriamycin plus $600 \mathrm{mg} / \mathrm{m}^{2}$ cyclophosphamide intravenously) every 3 weeks for 4 cycles, followed by taxol $\left(75 \mathrm{mg} / \mathrm{m}^{2}\right.$ docetaxel intravenously) every 3 weeks for 4 cycles, or goserelin acetate $3.6 \mathrm{mg}$ every 4 weeks with tamoxifen $20 \mathrm{mg}$ daily. Treatment continued for 24 weeks before surgery (Additional file 1).

All patients underwent breast magnetic resonance imaging (MRI) before the start of treatment and after the end of treatment before surgery. Additionally, during treatments (every 3 weeks for the NCT group, and every 4 weeks for the NET group), calliper measurement of the tumour size was performed. We determined the objective tumour response with every measurement method and assessed the response according to the modified RECIST [14]. Surgery was performed between the 24th and 26th week.

All immunohistochemical analyses of ER and Ki-67 expression were performed and reviewed at the central laboratory (Asan Medical Center, Seoul, South Korea). Ki-67 was stained with an antibody for MIB-1 for assessment, using a sample of the core biopsy prior to treatment and a surgery specimen after treatment. The Ki-67 index was calculated as the percentage ratio of Ki-67-positive cells to total cells.

\section{Outcomes}

The primary endpoint was the clinical response rate at 24 weeks, as determined using calliper and MRI measurements. We performed tumour measurements using callipers every 3 weeks for the NEC group and every 4 weeks for the NCT group, and MRI at day 0 and week 24. A clinical response included either a complete response (CR) or a partial response (PR) according to the Response Evaluation Criteria In Solid Tumour (RECIST) version 1.1 criteria. Secondary endpoints were the rate of pCR, the rate of breast conservation surgery, Ki-67 changes, the length of time to maximum response within a treatment period, and QoL, using the European Organisation for Research and Treatment of Cancer Quality of Life (EORTC QOL BR23) questionnaire. Adverse events were recorded at every patient visit and assessed according to the Common Terminology Criteria for Adverse Events Version 3.0.

\section{Statistical analysis}

The sample size was calculated based on the clinical response rate measured by MRI in each group, the NCT and NET groups, under the assumption that the effect of NET would be non-inferior to that of NCT. Sample sizes of 131 in each group achieve $80.055 \%$ power to detect a non-inferiority margin difference between the group proportions of -0.1500 (i.e. the lower limit of the twosided 95\% confidence interval (CI) for the difference in response rates between groups being $15 \%$ or less), when the MRI response rate of NCT and NET was estimated to be approximately $75 \%$. A total of 290 patients were required (145 per treatment group, allowing for a dropout rate of $10 \%$ in each group).

Because of enrolment failure, a total of 194 patients were enrolled until September 24, 2014. All data analysis was performed according to a pre-established analysis plan. A two-sided 95\% confidence interval for the difference in the incidence of morbidity was calculated to test for non-inferiority. As a non-inferiority design had been adopted, difference of the response rate between group and the $95 \%$ CI was calculated, using the index of NCT 
as the reference. NET would be considered to be noninferior to NCT when the lower limit of the $\mathrm{CI}$ is greater than -0.15 . Because of enrolment failure, a total of 194 patients were enrolled until September 24, 2014. To draw a comparison between the two groups, $t$ test for mean differences and chi-squared test for frequencies were used. A 2-sided $p<0.05$ was considered statistically significant. All analyses were conducted using SAS version 9.4 (SAS Institute; Cary, NC) and R version 3.6.1.

\section{Role of the funding source}

AstraZeneca participated in the funding of the study. The authors had complete access to the study data, and the corresponding author had the final responsibility for publication submission.

\section{Results}

A total of 187 patients from 7 participating centres were included and randomised. Seven patients in the NCT group and 5 patients in the NET group withdraw their consent. One patient in the NCT group was randomised but did not receive treatment. Therefore, a total 174 patients completed the scheduled treatment and were finally analysed (87 patients received NCT and 87 patients received NET; Fig. 1). Four patients in the NET group did not receive the surgery, of whom 3 patients showed PR and 1 patient showed stable disease (SD). The data of these 4 patients were excluded from the post-surgery data (pCR and rate of BCS) analysis (Fig. 1). Baseline characteristics were generally balanced between the treatment groups (Table 1). The median age was 42 years (range, 27-54). All patients were pre-menopausal, and $63 \%$ of patients had a normal body mass index. Sixty-nine percent of patients had a clinical T2 breast cancer, and $88.5 \%$ had a clinical N1 stage breast cancer. Ninety-four percent of patients had G1/2 breast cancer, and few patients (less than $5 \%$ ) had poorly differentiated (G3) tumour. The mean Ki-67 expression was not different between the two groups (26.3 for NCT vs. 26.7 for NET, $p=0.891$ ). Forty-eight percent of NCT patients and $61 \%$ of NET patients were expected to undergo mastectomy prior to the start of treatment $(p=0.142)$.

Tumour size decreased over 24 weeks in both treatment groups $(p>0.05)$. The clinical response rate measured using MRI was $83.7 \%$ (72 of 87 patients) with NCT and $52.9 \%$ (45 of 87 patients) with NET (estimated difference $30.8 \%$, 95\% CI 17.6-44.0, $p<0.001$ ). On callipers, the clinical response rate was $83.9 \%$ (73 of 87

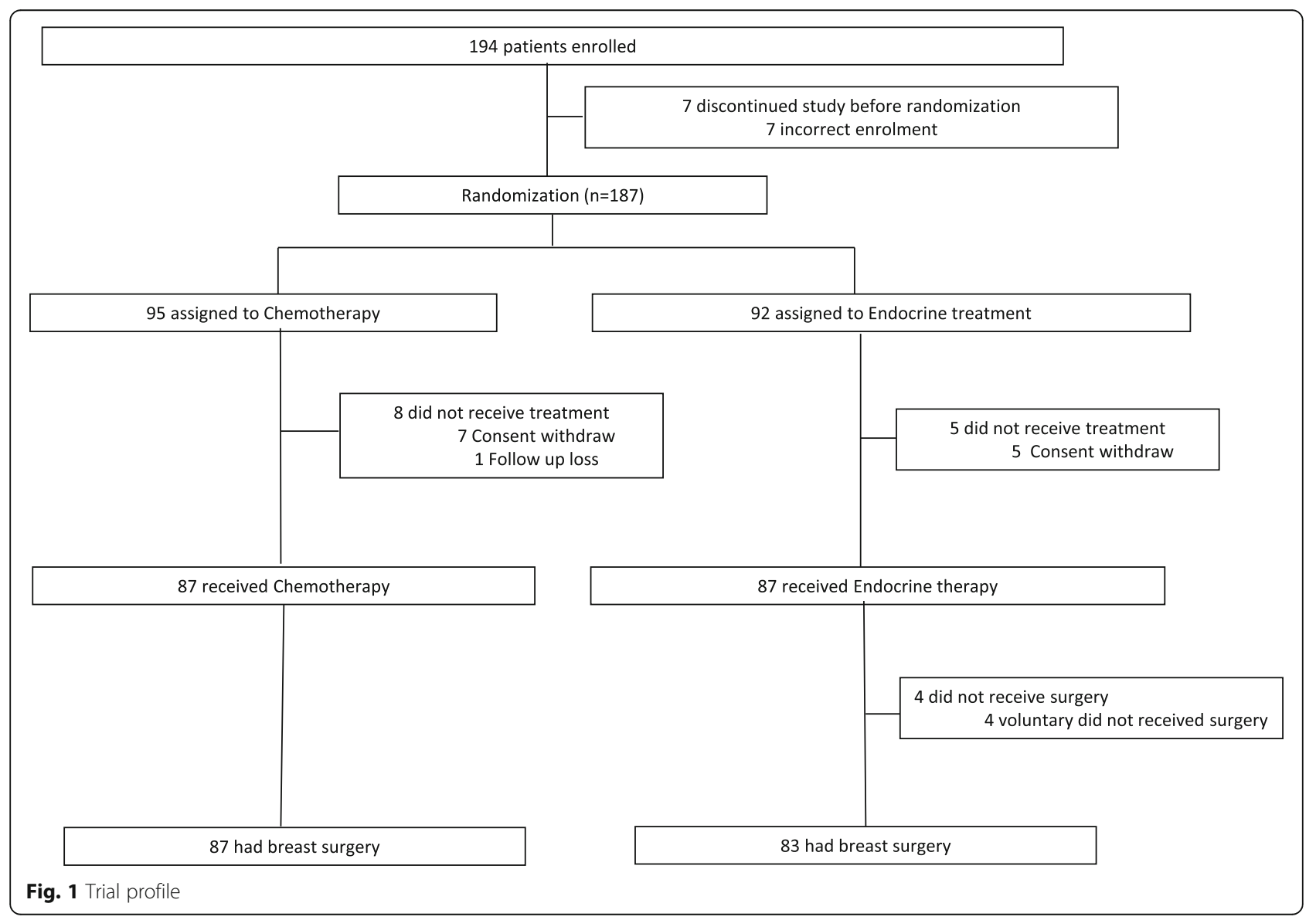


Table 1 Patient demographics and baseline tumour characteristics

\begin{tabular}{|c|c|c|c|}
\hline & Chemotherapy group $(\boldsymbol{n}=87)$ & Endocrine therapy group $(\boldsymbol{n}=87)$ & $\boldsymbol{p}$ value \\
\hline \multicolumn{4}{|l|}{ Age group at baseline(years) } \\
\hline Mean (SD) & $42.5 \pm 5.6$ & $41.5 \pm 5.8$ & 0.255 \\
\hline $20-29$ & $2(2.3 \%)$ & $2(2.3 \%)$ & \\
\hline $30-39$ & $20(23.0 \%)$ & $31(35.6 \%)$ & \\
\hline $40-49$ & $59(69.0 \%)$ & $50(59.8 \%)$ & \\
\hline $50-55$ & $6(5.7 \%)$ & $4(2.3 \%)$ & \\
\hline BMI $\left(\mathrm{kg} / \mathrm{m}^{2}\right)$ & & & 0.921 \\
\hline$<18.5$ & $5(5.7 \%)$ & $4(4.6 \%)$ & \\
\hline $18.5-24.9$ & $54(62.1 \%)$ & $56(64.4 \%)$ & \\
\hline $25-29.9$ & $24(27.6 \%)$ & $20(23.0 \%)$ & \\
\hline$\geq 30$ & $4(4.6 \%)$ & $7(8.0 \%)$ & \\
\hline Clinical T stage & & & 0.746 \\
\hline $\mathrm{T} 1$ & $13(14.9 \%)$ & $9(10.3 \%)$ & \\
\hline $\mathrm{T} 2$ & $58(66.7 \%)$ & $62(71.3 \%)$ & \\
\hline T3 & $16(18.4 \%)$ & $16(18.4 \%)$ & \\
\hline Clinical N stage & & & 0.808 \\
\hline N1 & 78 (89.7\%) & $76(87.4 \%)$ & \\
\hline N2 & $5(5.7 \%)$ & $5(5.7 \%)$ & \\
\hline N3 & $4(4.6 \%)$ & $6(6.9 \%)$ & \\
\hline Grade & & & 0.616 \\
\hline $\mathrm{G} 1 / 2$ & $52(59.8 \%)$ & $61(70.1 \%)$ & \\
\hline G3 & $3(3.4 \%)$ & $4(4.6 \%)$ & \\
\hline NA & $32(36.8 \%)$ & $22(25.3 \%)$ & \\
\hline Ki-67 expression (\%) & & & 0.891 \\
\hline$\leq 20 \%$ & 49 (56.3\%) & $48(55.2 \%)$ & \\
\hline$>20 \%$ & $36(41.4 \%)$ & $37(42.6 \%)$ & \\
\hline Unknown & $2(2.3 \%)$ & $2(2.3 \%)$ & \\
\hline Planned operation & & & 0.141 \\
\hline Mastectomy & $45(51.7 \%)$ & $53(60.6 \%)$ & \\
\hline Breast-conserving surgery & $42(48.3 \%)$ & 34 (39.1\%) & \\
\hline
\end{tabular}

Data are $n(\%)$, unless otherwise stated

patients) with NCT and $71.3 \%$ (62 of 87 patients) with NET (estimated difference 12.6\%, 95\% CI 0.4-24.9, $p=$ 0.046) (Table 2).

The conversion rate of breast-conserving surgery (BCS) with neoadjuvant treatment in patients who planned to have total mastectomy was $13.8 \%$ in the NCT group and $11.5 \%$ in the NET group, which was not significantly different $(p=0.531)$. Three NCT patients (3.4\%) and one NET patient (1.2\%) showed pCR (ypT0ypN0M0) $\quad(p<0.005)$. Twelve NCT patients (13.8\%) and 4 NET patients (4.9\%) had complete lymph node response $(p<0.005)$. However, Ki-67 expression change during the treatment period was not significantly different between the two groups $(p=0.114 ;-13.56$ for NCT and -7.49 for NET).

Nineteen NCT patients $(21.8 \%)$ had treatment-related grade 3 or worse adverse events, with no adverse effects in the NET group.

Exploratory subgroup analysis was performed. There was a difference of tumour response according to Ki-67 expression. For patients with a low Ki-67 expression ( $\leq$ $20 \%)$, the clinical response rate was $83.7 \%(41 / 49)$ in NCT, 60.4\% (29/48) in NET with MRI, and 69.4\% (34/ $49)$ in NCT, $83.3 \%(40 / 48)$ in NET with calliper. Among the high Ki-67 expression group (>20\%), the response rate was $81.1 \%(29 / 36)$ in NCT, $40.5 \%(15 / 37)$ in NET 
Table 2 Summary of clinical response from baseline to week 24

\begin{tabular}{|c|c|c|}
\hline \multirow[t]{2}{*}{ Clinical response } & \multicolumn{2}{|l|}{ Intention to treat population } \\
\hline & Chemotherapy group $(n=87)$ & Endocrine therapy group $(n=87)$ \\
\hline \multicolumn{3}{|l|}{$\mathrm{MRI}^{+}$} \\
\hline$C R$ & $14(16.3 \%)$ & $2(2.3 \%)$ \\
\hline PR & $58(67.4 \%)$ & $43(50.6 \%)$ \\
\hline$C R+P R$ & $72(83.7)$ & $45(52.9 \%)$ \\
\hline \multicolumn{3}{|l|}{ Calliper* } \\
\hline$C R$ & $27(31 \%)$ & $17(19.5 \%)$ \\
\hline$P R$ & $46(52.9 \%)$ & $45(51.5 \%)$ \\
\hline$C R+P R$ & $73(83.9 \%)$ & $62(71.3 \%)$ \\
\hline Pathologic response & Chemotherapy group $(n=87)$ & Endocrine therapy group $(n=83)$ \\
\hline $\mathrm{pCR}$ & $3(3.4 \%)$ & $1(1.2 \%)$ \\
\hline pCR (breast) & $5(5.7 \%)$ & $1(1.2 \%)$ \\
\hline pCR (axillary lymph node) & $12(13.8 \%)$ & $4(4.9 \%)$ \\
\hline
\end{tabular}

Data are $n$ (\%). $C R$ complete response, $P R$ partial response

${ }^{+}$Estimated difference $30.8 \%, 95 \% \mathrm{Cl} 17.6-44.0, p<0.001$

*Estimated difference $12.6 \%, 95 \% \mathrm{Cl} 0.4-24.9, p=0.046$

by MRI, and $77.8 \%(28 / 36)$ in NCT, $78.4 \%(29 / 37)$ in NET by calliper (Table 3, Fig. 2).

\section{Discussion}

During 24 weeks of neoadjuvant treatment, in premenopausal, hormone receptor-positive, HER2-negative, and lymph node-positive breast cancer patients, NCT achieved a significantly better clinical response rate than NET. The difference in response was even higher in patients with a highly proliferating tumour (Ki-67 expression $>20$ ). Neoadjuvant therapy was comparatively more effective in patients with low Ki67 (low, 60.4\%, vs. high, $40.5 \%$, by MRI) while chemotherapy was equally effective irrespectively of Ki67 (low, 81.1\%, vs. high, $83.7 \%$, by MRI).

This study is the first to compare NCT with NET (tamoxifen plus ovarian function suppression) in premenopausal breast cancer only. This study is also unique because the patients were all ER-positive/HER2-negative and lymph node-positive.

The primary finding of this study was that the response to NET was inferior to NCT in pre-menopausal ER-positive/HER2-negative subtype breast cancer. The pCR rate was also higher in the NCT group than in the NET group. However, this does not imply that NCT patients will have a better long-term survival outcome than NET patients. First, the changes in Ki-67 expression

Table 3 Summary of clinical response from baseline to week 24

\begin{tabular}{|c|c|c|c|c|c|c|}
\hline & \multicolumn{2}{|l|}{ Treatment group } & \multicolumn{2}{|l|}{ Low Ki subgroup } & \multicolumn{2}{|l|}{ High Ki subgroup } \\
\hline & $\begin{array}{l}\text { Chemotherapy arm } \\
(n=87)\end{array}$ & $\begin{array}{l}\text { Endocrine therapy } \\
\operatorname{arm}(n=87)\end{array}$ & $\begin{array}{l}\text { Chemotherapy arm } \\
(n=49)\end{array}$ & $\begin{array}{l}\text { Endocrine therapy } \\
\operatorname{arm}(n=48)\end{array}$ & $\begin{array}{l}\text { Chemotherapy arm } \\
(n=36)\end{array}$ & $\begin{array}{l}\text { Endocrine therapy } \\
\operatorname{arm}(n=37)\end{array}$ \\
\hline \multicolumn{7}{|c|}{ Best overall tumour response } \\
\hline \multicolumn{7}{|l|}{ MRI } \\
\hline$C R$ & $14(16.3 \%)$ & $2(2.3 \%)$ & $6(12.2 \%)$ & $1(2.1 \%)$ & 7 (19.4\%) & $1(2.7 \%)$ \\
\hline PR & $58(67.4 \%)$ & $43(50.6 \%)$ & $35(71.4 \%)$ & $28(58.3 \%)$ & $22(61.1 \%)$ & $14(37.8 \%)$ \\
\hline $\begin{array}{l}\mathrm{CR}+ \\
\mathrm{PR}\end{array}$ & $72(83.7)$ & $45(52.9 \%)$ & $41(83.7 \%)$ & $29(60.4 \%)$ & $29(81.1 \%)$ & $15(40.5 \%)$ \\
\hline \multicolumn{7}{|l|}{ Calliper } \\
\hline$C R$ & $27(31 \%)$ & $17(19.5 \%)$ & $12(24.5 \%)$ & $10(20.8 \%)$ & $8(22.2 \%)$ & $13(35.5 \%)$ \\
\hline PR & $46(52.9 \%)$ & 45 (51.5\%) & 22 (44.9\%) & $30(62.5 \%)$ & $20(55.6 \%)$ & $16(43.2 \%)$ \\
\hline $\begin{array}{l}C R+ \\
P R\end{array}$ & $73(83.9 \%)$ & $62(71.3 \%)$ & $34(69.4 \%)$ & $40(83.3 \%)$ & $28(77.8 \%)$ & $29(78.4 \%)$ \\
\hline
\end{tabular}



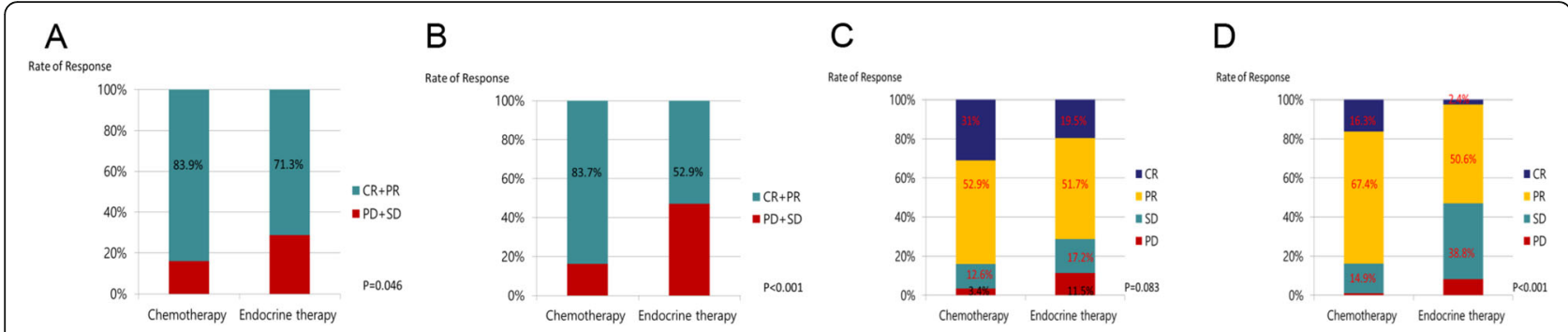

Fig. 2 Response rate according to treatments. a Clinical response measure by calliper. b Clinical response measure by MRI. c Detailed clinical response measure by calliper. $\mathbf{d}$ Detailed clinical response measure by MRI

were not different between the two groups. In addition, NET patients could receive adjuvant chemotherapy in most cases especially when the response was poor (data not shown). Long-term follow-up of the two patient groups is warranted.

Considering pCR and/or clinical response is not a reliable surrogate endpoint for survival, and the most important role of neoadjuvant systemic therapy in ER-positive/HER2negative breast cancer is expanding the pool of potential BCS candidates by downstaging tumours and permitting BCS in patients who would otherwise require mastectomy [18]. In our study, although NCT led to a better clinical response, the BCS rate was not different between the two groups. Therefore, for the purpose of enabling BCS for mastectomy candidates, NCT might not be a better option than NET.

A potential advantage of neoadjuvant therapy is avoidance of axillary lymph node dissection in patients who have had negative conversion of tumour-positive lymph nodes. NCT downstages axillary nodes in 20 to $40 \%$ of patients, and these rates are even higher $(>50 \%)$ in HER2positive patients given anti-HER2 therapy [19-21]. Two recent prospective trials (ACOSOG Z1071 and SENTINA) reported that the false-negative rate was reasonably low when a dual tracer was used and 3 or more sentinel nodes were harvested [22, 23]. Kang et al. showed that in breast cancer patients who had axillary lymph node conversion from clinically positive to negative following NCT, a sentinel lymph node biopsy-guided axillary operation had similar rates of axillary and distant recurrence with axillary lymph node dissection without sentinel node biopsy [24]. Our study showed that for the purpose of avoiding lymph node dissection, NCT can be better than NET because the CR in lymph nodes was significantly higher in the NCT group than in the NET group (13.8\% vs. $4.6 \%)$. However, further study is needed as to whether complete axillary lymph node dissection has survival benefit and should be a mandatory procedure for patients who have metastasis in a limited number of axillary lymph nodes after NCT or NET.

Most NET studies in breast cancer have focused on post-menopausal women $[12,13,25,26]$. Few data are available on NET in pre-menopausal women. Some studies have shown that NET could be effective in wellselected pre-menopausal patients [17, 27]. GEICAM has reported randomised phase II results of chemotherapy versus exemestane in pre- and post-menopausal women [28]. Although the sample size was small, the response rate was higher for chemotherapy than for endocrine therapy in pre-menopausal patients, which was consistent with our study. The two therapies were comparable in post-menopausal women and those with a low baseline Ki-67.

The optimal duration of NET is not well defined. Most previous NET studies applied from 3 to 6 months of therapy. In a study by Llombart-Cussac et al. [26], 37\% of patients achieved the maximal response beyond 6 months. Carpenter et al. showed that median time to achieve BCS (in those who responded) was 7.5 months [29]. Notably, 62\% of panellists at St Gallen 2013 were in favour of continuing NET until a maximal response [30]. In our study, the patients received 6 months of NET. If we had treated to the maximal response for these patients, the response might have been better.

For ER-positive/HER2-negative subtype breast cancer, objective response determination is an issue. $\mathrm{pCR}$, which is the only definite response criteria in neoadjuvant therapy, is highly uncommon; furthermore, it is not a surrogate endpoint for survival in this subtype [31]. We evaluated the clinical response using MRI and callipers. Even though MRI is a useful method for measuring residual tumour size after neoadjuvant treatment, it is not well correlated with pathologic size in ER-positive, HER2-negative tumours [32]. In our study, there was also a large discrepancy between MRI size and pathologic tumour size in the NCT group (data not shown). The difference in response pattern in the tumour between endocrine therapy and chemotherapy makes an objective response determination and comparison difficult and somewhat imbalanced.

There are some limitations in this study. First, the small sample size which was not satisfied with predefined number was included. Second, clinical response or $\mathrm{pCR}$ is not a predictor of prognosis in ER-positive 
and Her2-negative subtypes. This data cannot demonstrate the survival benefit of NCT compared with NET. Third, we did not include the arm of aromatase inhibitor as a NET treatment. In a study that compared neoadjuvant GnRH analogue plus tamoxifen and $\mathrm{GnRH}$ analogue plus anastrozole in pre-menopausal women, the clinical response was better in the anastrozole group [17]. Because we did not consider an aromatase inhibitor, a question remains concerning the response comparison between chemotherapy versus ovarian function suppression with aromatase inhibitor in pre-menopausal women. Lastly, multigene assays were not evaluated.

Although, in our result for the primary endpoint, NET showed a worse clinical response than NCT, NET cannot be excluded as a clinical option, because (1) the BCS rate was comparable, (2) no difference was observed in Ki-67 change with the therapies, and (3) the adverse event was much smaller in the NET group. This finding indicates that we can recommend NET in clinical practice in ER-positive/HER2-negative breast cancer with low Ki-67 expression, intermediate/low grade tumour, clinically lymph node-negative patients, and for patients with large tumours where BCS is not feasible or equivocal. It is recommended to perform a multigene assay (e.g. Oncotype DX, Mammprint) prior to the start of NET to confirm whether chemotherapy is needed for the individual patient.

\section{Conclusion}

In pre-menopausal breast cancer patients, 24 weeks of NCT showed a better clinical response than NET except for in a subgroup with low Ki-67 or low grade cancer. However, the breast conservation rate and the Ki-67 change were not different. There may be an issue concerning accurate size measurements with MRI in both groups. Clinicians should be aware of the potential benefits and disadvantages of both treatments. Use of NET in pre-menopausal women requires further discussion.

\section{Supplementary information}

Supplementary information accompanies this paper at https://doi.org/10. 1186/s13058-020-01288-5.

Additional file 1. Drug administration and Statistics.

\footnotetext{
Abbreviations

NET: Neoadjuvant endocrine therapy; NCT: Neoadjuvant chemotherapy; TAILORx: The Trial Assigning Individualised Options for Treatment; RxPonder: Rx for positive node, endocrine responsive breast cancer: MINDACT: Microarray In Node negative Disease may Avoid Chemotherapy; NNBC-3: Node Negative Breast Cancer III Study; ASCO: American Society of Clinical Oncology; SOFT: Suppression of Ovarian Function Trial; TEXT: Tamoxifen and Exemestane Trial; pCR: Pathologic complete response; GnRHa: Gonadotropin-releasing hormone agonist; RECIST: Response Evaluation Criteria In Solid Tumours; IHC: Immunohistochemistry; PEPI: Preoperative endocrine prognostic index; CPS-EG score: Clinicpathologic stage-oestrogen receptor and tumour grade score
}

\section{Acknowledgements}

This research was conducted with financial and medicinal product support from AstraZeneca Korea Ltd.

\section{Authors' contributions}

SA and HK designed the study. HK and WH drafted the manuscript, and HK wrote the original protocol for the study. All authors participated in the design of the study. HK filed for the ethical approvals from KFDA and registered the trial on ClinicalTrials.gov. $\mathrm{HK}^{9}$ performed the statistical analysis. WN conceived the study and participated in its design. GG carried out the immunoassays. EL, YJ, LK, WH, and SN were involved in the study design and inclusion of patients in this trial. All authors read and approved the final manuscript.

\section{Funding}

This work was supported by the AstraZeneca Korea.

\section{Availability of data and materials}

The datasets analysed during the current study are available from the corresponding authors on reasonable request.

\section{Ethics approval and consent to participate}

This study protocol has been approved by the Korea Food and Drug Administration (KFDA), as well as the institutional review board of every trial centre, and was conducted in accordance with the Declaration of Helsinki, Good Clinical Practice, and the applicable local regulatory requirements on bioethics. Written informed consent was obtained from all participants.

\section{Consent for publication}

Not applicable

\section{Competing interests}

All authors declare that they have no competing interests.

\section{Author details}

${ }^{1}$ Department of Surgery, University of Ulsan College of Medicine, Asan Medical Center, 88 Olympic ro 43 gil, song pa gu, Seoul 138-736, South Korea. ${ }^{2}$ Department of Surgery, Korea Cancer Center Hospital, Korea Institute of Radiological and Medical Sciences, Seoul, South Korea. ${ }^{3}$ Department of Surgery, Center for Breast Cancer, Research and Institute and Hospital, National Cancer Center, Goyang, South Korea. ${ }^{4}$ Department of Surgery, School of Medicine, Ajou University, Suwon, South Korea. ${ }^{5}$ Division of Breast and Endocrine Surgery, Hallym Sacred Heart Hospital, College of Medicine, Hallyum University, Anyang, South Korea. ${ }^{6}$ Department of Surgery and Cancer Research Institute, College of Medicine, Seoul National University, Seoul, South Korea. 'Department of Surgery, Samsung Medical Center, School of Medicine, Sungkyunkwan University, Seoul, South Korea. ${ }^{8}$ Department of Pathology, University of Ulsan College of Medicine, Asan Medical Center, Seoul, South Korea. ${ }^{9}$ Department of Preventive Medicine, University of Ulsan College of Medicine, Asan Medical Center, Seoul, South Korea.

Received: 7 December 2019 Accepted: 3 May 2020

Published online: 27 May 2020

\section{References}

1. Ahn SH, Son BH, Kim SW, Kim Sl, Jeong J, Ko S-S, Han W. Poor outcome of hormone receptor-positive breast Cancer at very young age is due to tamoxifen resistance: nationwide survival data in Korea - a report from the Korean Breast Cancer Society. J Clin Oncol. 2007;25(17):2360-8.

2. Swain SM, Jeong J-H, Wolmark N. Amenorrhea from breast cancer therapy — not a matter of dose. N Engl J Med. 2010;363(23):2268-70.

3. Swain SM, Jeong J-H, Geyer CEJ, Costantino JP, Pajon ER, Fehrenbacher L, Atkins JN, Polikoff J, Vogel VG, Erban JK, et al. Longer therapy, iatrogenic amenorrhea, and survival in early breast cancer. N Engl J Med. 2010;362(22): 2053-65.

4. Jung M, Shin HJ, Rha SY, Jeung HC, Hong S, Moon YW, Kim HS, Oh KJ, Yang WI, Roh JK, et al. The clinical outcome of chemotherapy-induced amenorrhea in premenopausal young patients with breast cancer with long-term follow-up. Ann Surg Oncol. 2010;17(12):3259-68. 
5. Goncalves R, Bose R. Using multigene tests to select treatment for earlystage breast cancer. J Natl Compr Cancer Netw. 2013;1 (2):174-82.

6. Harbeck N, Schmitt M, Meisner C, Friedel C, Untch M, Schmidt M, Sweep CGJ, Lisboa BW, Lux MP, Beck T, et al. Ten-year analysis of the prospective multicentre Chemo-NO trial validates American Society of Clinical Oncology (ASCO)-recommended biomarkers UPA and PAl-1 for therapy decision making in node-negative breast cancer patients. Eur J Cancer. 2013;49(8): 1825-35.

7. Sparano JA, Gray RJ, Makower DF, Pritchard KI, Albain KS, Hayes DF, Geyer CE Jr, Dees EC, Goetz MP, Olson JA Jr, et al. Adjuvant chemotherapy guided by a 21-gene expression assay in breast cancer. N Engl J Med. 2018;379(2): $111-21$.

8. Cardoso F, van't Veer L, Bogaerts J, Slaets L, Viale G, Delaloge S, Pierga JY, Brain E, Causeret S, DeLorenzi M, et al. 70-gene signature as an aid to treatment decisions in early-stage breast cancer. N Engl J Med. 2016;375(8): 717-29.

9. Albain KS, Barlow WE, Shak S, Hortobagyi GN, Livingston RB, Yeh IT, Ravdin P, Bugarini R, Baehner FL, Davidson NE, et al. Prognostic and predictive value of the 21-gene recurrence score assay in postmenopausal women with node-positive, oestrogen-receptor-positive breast cancer on chemotherapy: a retrospective analysis of a randomised trial. Lancet Oncol. 2010;11(1):55-65.

10. Lim E, Winer EP. Adjuvant chemotherapy in luminal breast cancers. Breast. 2011;20(Supplement 3):S128-31.

11. Chang J, Lee A, Lee J, Lim W, Sung SH, Moon B-I. Correlation between the molecular subtype of breast cancer and the in vitro adenosine triphosphate-based chemosensitivity assay. J Korean Surg Soc. 2013;84(6): 313-20.

12. Gv M, Blohmer JU, Costa SD, Denkert C, Eidtmann H, Eiermann W, Gerber B, Hanusch C, Hilfrich J, Huober J, et al. Response-guided neoadjuvant chemotherapy for breast cancer. J Clin Oncol. 2013;31(29):3623-30.

13. Ellis MJ, Coop A, Singh B, Tao Y, Llombart-Cussac A, Jänicke F, Mauriac L, Quebe-Fehling E, Chaudri-Ross HA, Evans DB, et al. Letrozole inhibits tumor proliferation more effectively than tamoxifen independent of HER1/2 expression status. Cancer Res. 2003;63(19):6523-31.

14. Smith IE, Dowsett M, Ebbs SR, Dixon JM, Skene A, Blohmer J-U, Ashley SE, Francis S, Boeddinghaus I, Walsh G. Neoadjuvant treatment of postmenopausal breast cancer with anastrozole, tamoxifen, or both in combination: the immediate preoperative anastrozole, tamoxifen, or combined with tamoxifen (IMPACT) multicenter double-blind randomized trial. J Clin Oncol. 2005;23(22):5108-16.

15. Cataliotti L, Buzdar AU, Noguchi S, Bines J, Takatsuka Y, Petrakova K, Dube P, Tosello de Oliveira C. Comparison of anastrozole versus tamoxifen as preoperative therapy in postmenopausal women with hormone receptorpositive breast cancer. Cancer. 2006;106(10):2095-103.

16. Semiglazov VF, Semiglazov W, Dashyan GA, Ziltsova EK, Ivanov VG, Bozhok AA, Melnikova OA, Paltuev RM, Kletzel A, Berstein LM. Phase 2 randomized trial of primary endocrine therapy versus chemotherapy in postmenopausal patients with estrogen receptor-positive breast cancer. Cancer. 2007;110(2): 244-54

17. Masuda N, Sagara Y, Kinoshita T, Iwata H, Nakamura S, Yanagita Y, Nishimura $R$, Iwase $H$, Kamigaki S, Takei H, et al. Neoadjuvant anastrozole versus tamoxifen in patients receiving goserelin for premenopausal breast cancer (STAGE): a double-blind, randomised phase 3 trial. Lancet Oncol. 2012;13(4): 345-52.

18. Hage AN, Capriccioso C, Brennan J, Heiden B, Zheutlin A, Sabel MS. Impact of neoadjuvant chemotherapy on surgical outcomes among patients with hormone receptor positive breast cancer. J Surg Oncol. 2017;116(6):665-70.

19. Kuerer HM, Sahin AA, Hunt KK, Newman LA, Breslin TM, Ames FC, Ross MI, Buzdar AU, Hortobagyi GN, Singletary SE. Incidence and Impact of documented eradication of breast cancer axillary lymph node metastases before surgery in patients treated with neoadjuvant chemotherapy. Ann Surg. 1999;230(1):72.

20. Baselga J, Bradbury I, Eidtmann H, Di Cosimo S, de Azambuja E, Aura C, Gómez H, Dinh P, Fauria K, Van Dooren V, et al. Lapatinib with trastuzumab for HER2-positive early breast cancer (NeoALTTO): a randomised, open-label, multicentre, phase 3 trial. Lancet. 2012;379(9816):633-40.

21. Kim HS, Shin MS, Kim CJ, Yoo SH, Yoo TK, Eom YH, Chae BJ, Song BJ. Improved model for predicting axillary response to neoadjuvant chemotherapy in patients with clinically node-positive breast cancer. J Breast Cancer. 2017;20(4):378-85.
22. Boughey JC, Suman VJ, Mittendorf EA, et al. Sentinel lymph node surgery after neoadjuvant chemotherapy in patients with node-positive breast cancer: the acosog z1071 (alliance) clinical trial. JAMA. 2013;310(14):1455-61.

23. Kuehn T, Bauerfeind I, Fehm T, Fleige B, Hausschild M, Helms G, Lebeau A, Liedtke C, Gv M, Nekljudova V, et al. Sentinel-lymph-node biopsy in patients with breast cancer before and after neoadjuvant chemotherapy (SENTINA): a prospective, multicentre cohort study. Lancet Oncol. 2013;14(7):609-18.

24. Kang Y-J, Han W, Park S, You JY, Yi HW, Park S, Nam S, Kim JH, Yun KW, Kim $\mathrm{HJ}$, et al. Outcome following sentinel lymph node biopsy-guided decisions in breast cancer patients with conversion from positive to negative axillary lymph nodes after neoadjuvant chemotherapy. Breast Cancer Res Treat. 2017; 166(2):473-80.

25. Palmieri C, Cleator S, Kilburn LS, Kim SB, Ahn S-H, Beresford M, Gong G, Mansi J, Mallon E, Reed S, et al. NEOCENT: a randomised feasibility and translational study comparing neoadjuvant endocrine therapy with chemotherapy in ER-rich postmenopausal primary breast cancer. Breast Cancer Res Treat. 2014;148(3):581-90.

26. Llombart-Cussac A, Guerrero Á, Galán A, Carañana V, Buch E, RodríguezLescure Á, Ruiz A, Fuster Diana C, Guillem Porta V. Phase II trial with letrozole to maximum response as primary systemic therapy in postmenopausal patients with ER/PgR $[+]$ operable breast cancer. Clin Transl Oncol. 2012;14(2):125-31.

27. Barbie TU, Ma C, Margenthaler JA. Management of premenopausal women with neoadjuvant endocrine therapy: a single-institution experience. Ann Surg Oncol. 2015;22(12):3861-5.

28. Alba E, Calvo L, Albanell J, De la Haba JR, Arcusa Lanza A, Chacon Jl, Sanchez-Rovira P, Plazaola A, Lopez Garcia-Asenjo JA, Bermejo B, et al. Chemotherapy (CT) and hormonotherapy ( $\mathrm{HT}$ ) as neoadjuvant treatment in luminal breast cancer patients: results from the GEICAM/2006-03, a multicenter, randomized, phase-II study. Ann Oncol. 2012;23(12):3069-74.

29. Carpenter R, Doughty JC, Cordiner C, Moss N, Gandhi A, Wilson C, Andrews C, Ellis G, Gui G, Skene Al. Optimum duration of neoadjuvant letrozole to permit breast conserving surgery. Breast Cancer Res Treat. 2014;144(3):56976.

30. Goldhirsch A, Winer EP, Coates AS, Gelber RD, Piccart-Gebhart M, Thürlimann B, Senn HJ, Albain KS, André F, Bergh J, et al. Personalizing the treatment of women with early breast cancer: highlights of the St Gallen International Expert Consensus on the Primary Therapy of Early Breast Cancer 2013. Ann Oncol. 2013;24(9):2206-23.

31. Cortazar P, Zhang L, Untch M, Mehta K, Costantino JP, Wolmark N, Bonnefoi $\mathrm{H}$, Cameron D, Gianni L, Valagussa P, et al. Pathological complete response and long-term clinical benefit in breast cancer: the CTNeoBC pooled analysis. Lancet. 2014;384(9938):164-72.

32. Moon H-G, Han W, Ahn SK, Cho N, Moon WK, Im S-A, Park IA, Noh D-Y. Breast cancer molecular phenotype and the use of HER2-targeted agents influence the accuracy of breast MRI after neoadjuvant chemotherapy. Ann Surg. 2013;257(1):133-7.

\section{Publisher's Note}

Springer Nature remains neutral with regard to jurisdictional claims in published maps and institutional affiliations.

Ready to submit your research? Choose BMC and benefit from:

- fast, convenient online submission

- thorough peer review by experienced researchers in your field

- rapid publication on acceptance

- support for research data, including large and complex data types

- gold Open Access which fosters wider collaboration and increased citations

- maximum visibility for your research: over $100 \mathrm{M}$ website views per year

At BMC, research is always in progress.

Learn more biomedcentral.com/submissions 